\title{
Retroperitoneal Teratoma in an Infant
}

Aditya Pratap Singh, Mahendra Jangid, DP Morya, Arun Gupta, Pradeep Gupta,

Mohit Sharma', Raj Laxmi Pardeshi'

From the Departments of Pediatric Surgery, Cardio-Vascular and Thoracic Surgery',

Gynae and Obstetrics ${ }^{2}$; S.M.S. Medical College, Jaipur, India.

\section{Abstract:}

Retroperitoneal teratoma in infancy is exceedingly rare. Progressive enlargement of the abdomen and the presence of an intra-abdominal mass is the most common clinical feature. They constitute third most frequent type of retroperitoneal neoplasm seen in infant and children after Wilm's tumor and neuroblastoma. We report here a case of giant retroperitoneal teratoma in an infant. A 5 month old female child was admitted with complain of abdominal distension. Abdominal CT scan revealed a tumor suggestive of retroperitoneal teratoma. Intraoperative finding revealed that tumor was compressing the atrophic right kidney. Resection of tumor with right nephrectomy was done successfully.

Key words: Retroperitoneal Neoplasms, Neuroblastoma, Teratoma, Wilms Tumor, Nephrectomy, Infant.

\section{Introduction}

Germ cell tumor is congenital tumor containing derivatives of all the three germ layers. Germ cell tumors arise due to aberrant migration of germ cells from yolk sac during fetal development. They are frequently seen in gonads [1]. Extra gonadal primary teratoma is usually encountered in infants and children. The involvement of extra-gonadal sites in decreasing order of frequency is mediastinum, sacrococcygeal region, retro peritoneum, pineal gland [1-4]. An incidence of teratoma is reported to be $6-11 \%$ of primary retroperitoneal tumors, with $60 \%$ occurring in children less than 15 years old, $70 \%$ of which occurs in infant. Here we present a huge retroperitoneal teratoma in 5 month old infant.

\section{Case Report}

Five month old female was admitted with complain of slowly progressive abdominal distension since 1 month. This was accompanied by frequent episodes of vomiting, poor feeding and respiratory distress. There was no notable family or past medical history. Her physical examination was significant for ill-defined and firm abdominal mass in right lumbar and iliac area. The remainder of the examination, including a thorough neurological and cardiovascular examination, was unremarkable.

Laboratory studies demonstrated: hemoglobin $13 \mathrm{gm} \%$, total leukocyte count 15800/cu mm with differential of $17 \%$ polymorphs and $71 \%$ lymphocytes. Serum electrolytes, renal function

Corresponding Author: Dr. Aditya Pratap Singh

Email: dr.adisms@gmail.com

Received: June 23,2014 | Accepted: July 30, 2014 | Published Online: August 25, 2014

This is an Open Access article distributed under the terms of the Creative Commons Attribution License (creativecommons.org/licenses/by/3.0)

Conflict of interest: None declared | Source of funding: Nil | DOl: http://dx.doi.org/10.17659/01.2014.0079 
test and liver function test were in normal limits. $X$ ray abdomen erect showed the bowel gas shadow pushed to the left side of abdomen by a large space occupying lesion. Ultrasound abdomen was suggestive of right sided Wilm's tumor of kidney. CECT abdomen revealed large right sided suprarenal mass $196 \times 120 \times 139 \mathrm{~mm}$ size showing multiloculated, cystic, calcification, fat and vascularity in retroperitoneum suggestive of terato-carcinoma. DTPA scan showed right sided nonfunctioning and atrophic kidney while left normal functioning kidney.

Intraoperative finding revealed a large retroperitoneal tumor consisting of solid and cystic area occupying mainly the right and central abdomen [Fig.1]. Tumor was adherent to surrounding structure and compressing the right kidney from upper side. Right kidney was atrophic and small in size. There was no infiltration of major vessels and no significant lymphadenopathy was present. Complete radical excision of tumor was done and closure done in layers [Fig.2]. The child was discharged on the $7^{\text {th }}$ postoperative day on full oral feed. Histopathological examination from tumor showed feature of immature teratoma grade 1.

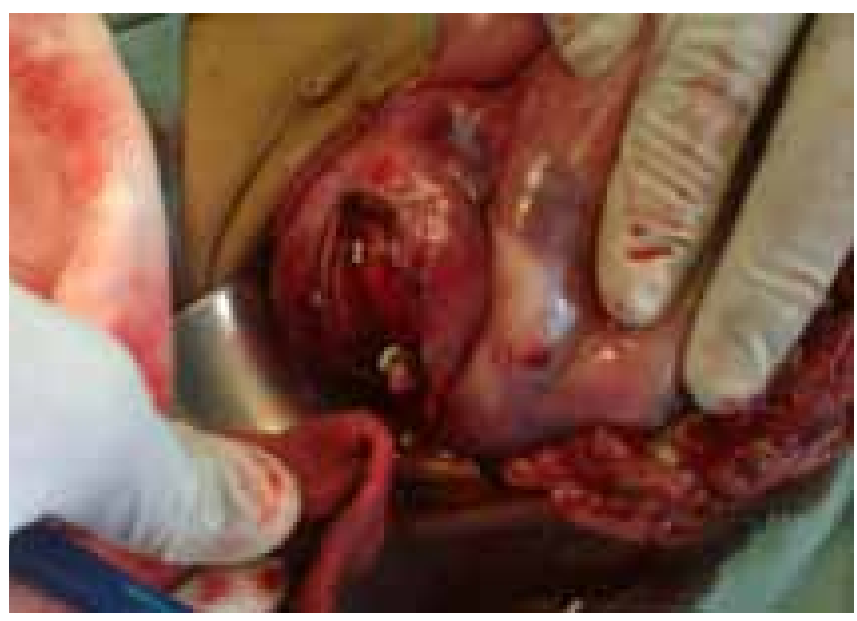

Fig.1: Operative photo showing incision on retroperitoneal mass.

\section{Discussion}

Germ cell tumor contains the derivatives of all the three germ layers [1-4]. Teratomas are often asymptomatic as retro-peritoneal space is extensive enough to allow for their free growth. They rarely present with acute syndrome leading to peritonitis, intestinal obstruction or renal colic. Retroperitoneal teratoma comprise $3.5-4 \%$ of all germ cell tumor in children and $1-11 \%$ of primary retro peritoneal neoplasm [5]. Antenatal diagnosed teratoma have higher incidence of malignancy than those in older children.

Patients usually present with abdominal distension or a palpable mass. An accurate diagnosis of a teratoma cannot be made on clinical basis. Radiological features include presence of calcification, teeth and fat, however calcification cannot be considered an indicator of a benign tumor since $12.5 \%$ of calcified tumor are malignant. Ultrasound of abdomen is usually the first imaging modality used in pediatric abdominal mass.

CT scan is useful to delineate the extent of the disease in retro-peritoneum and its relationship to

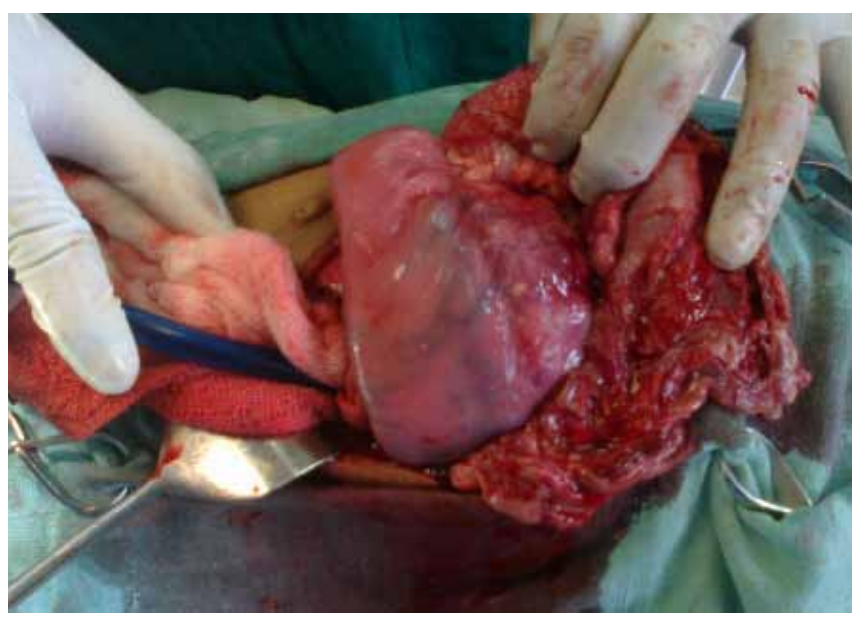

Fig.2: Operative photo showing retroperitoneal mass. 
major vessels. Internal homogeneous, fat density, cyst formation and calcification are considered important predictor of a benign retro peritoneal teratoma on CT scan. Some authors advocated angiography, inferior venography and needle biopsy for the accurate diagnosis of teratoma. Serum alpha-feto protein level is good indicator for diagnosis and assessing the recurrence of tumor.

Complete excision of tumor offers the best chance of cure [6]. Malignancy is uncommon in retroperitoneal teratoma hence non mutilating excision is possible and should be attempted [7]. Prognosis is generally good and curative if the tumor is completely removed. Most important prognostic factor is complete removal, however as it is possible that histologically mature tumor may take a malignant clinical course. Careful follow up is necessary in these patients.

In our case, age of the patient was 5 month and retroperitoneal teratoma is very unusual in this age group and at this site. Postoperative recovery was uneventful. At present there is no sign of recurrence of malignancy at follow up of 1 year. However such kind of long follow-up of this kind of rare case in Indian scenario is unusual.

\section{Conclusion}

Retroperitoneal teratoma can also present in infantile and juvenile age group. This is the alarming situation for pediatricians and pediatric surgeons. However early management like surgery can give good outcome to the patient.

\section{References}

1. Horton Z, Schlatter M, Schultz S: Pediatric germ cell tumors. Surg Oncol 2007;16(3):205-213.

2. McKenney JK, Heerema-McKenney A, Rouse RV. Extra-gonadal germ cell tumors: a review with emphasis on pathologic features, clinical prognostic variables, and differential diagnostic considerations. Adv Anat Pathol 2007; 14(2):6992.

3. Ueno T, Tanaka YO, Nagata M, Tsunoda H, Anno I, Ishikawa S, Kawai K, Itai Y. Spectrum of germ cell tumors: from head to toe. Radiographics 2004;24:387-404.

4. Schmoll HJ. Extragonadal germ cell tumors Ann Oncol 2002; 13:S265-S272.

5. Nguyen CT, Kratovil T, Edwards MJ. Retroperitoneal teratoma presenting as an abscess in childhood. J Pediatr Surg 2007;42:21-23.

6. Amit C, Samir M, Ashish W, Tandon RK, Wakhlu AK. Retroperitoneal Teratomas in Children. Indian J Pediatr 2006;73:221-223.http://www. diagnosticpathology.org/pubmed/1656791. Accessed on June 23, 2014.

7. Jones NM, Kiely EM. Retroperitoneal teratomaspotential for surgical misadventure. J Pediatr Surg 2008;43:184-186. 